\title{
Impacto en la imagen corporativa ante la inclusión de comunidades LGBT en tiendas retail en el Perú
}

\section{Impact on corporate image in the face of inclusion of LGBT communities in retail stores in Peru}

Recibido: 07/09/2020

Aceptado: $15 / 05 / 2021$

Publicado: 30/06/2021
Tania Karen Santa Cruz Jaime u201311872@upc.edu.pe https://orcid.org/0000-0001-5526-7528

Miguel Eduardo Antezana Corrieri pcimmant@upc.edu.pe https://orcid.org/0000-0002-7372-9420

Universidad Peruana de Ciencias Aplicadas (Perú)

Resumen: Este artículo analiza el impacto en la imagen corporativa como producto de la inclusión de miembros de la comunidad de lesbianas, gays, bisexuales y transgénero (LGBT) en un grupo de comercios retail en el Perú. Mediante una metodología mixta, se buscó determinar cuáles son las percepciones de los clientes al ver o interactuar con personas de dichas comunidades al momento de visitar tiendas especializadas en ropa; y cuál es la influencia de esa interacción en la formación de la imagen corporativa de esos establecimientos comerciales. Ante la discriminación en espacios laborales por la orientación sexual de sus trabajadores y la ausencia de políticas públicas en favor de las comunidades LGBT, este estudio muestra que la no inclusión se basa más en prejuicios o estereotipos que en consecuencias directas negativas y que, comunicacionalmente, no hay un perjuicio en la imagen corporativa de las empresas en los públicos con los que interactúa. 
Palabras clave: Minorías sexuales, LGBT, Discriminación, Inclusión laboral, Discriminación cultural, Imagen corporativa.

Abstract: This article analyzes the impact on corporate image as a product of the inclusion of members of the lesbian, gay, bisexual and transgender (LGBT) community in a group of retail stores in Peru. Through a mixed methodology, it was sought to determine what are the perceptions of customers when seeing or interacting with people from these communities when visiting specialized clothing stores; and what is the influence of this interaction in the formation of the corporate image of these commercial establishments. Faced with discrimination in work spaces due to the sexual orientation of their workers and the absence of public policies in favor of LGBT communities, this study shows that non-inclusion is based on prejudices or stereotypes that lead to negative direct consequences and that, communicationally, not there is damage to the corporate image of companies in the public with which it interacts.

Key words: Sexual minorities, LGBT, Discrimination, Labor inclusion, Cultural discrimination, Corporate image.

\section{Introducción}

El movimiento social de lesbianas, gays, bisexuales y transgénero es reconocido por sus siglas LGBT y se refiere a un grupo de personas con orientaciones homosexuales (Mejía \& Almanza, 2010). Por su parte, Sánchez (2017) señala que el movimiento LGBT pertenece a la clasificación de los nuevos movimientos sociales, ya que sus acciones colectivas están dirigidas al reconocimiento de los derechos de la comunidad homosexual, a través de la exigencia de igualdad de derechos de la sociedad heterosexual. Pizer (2012, como se citó en Sabharwal et. al., 2018) señala que esta población ha sido históricamente marginada a través de la negación sistemática de protecciones y beneficios legales. La histórica falta de aceptación de los estilos de vida de las personas LGBT por parte de la cultura, en general, los ha impulsado a buscar espacios sociales en donde puedan conocer a otros y expresar su sexualidad libremente (Barret \& Pollack, 2005). 
Para Meccia (2011) "la mezcla de visibilidad legítima de los gays más la pertenencia económico-social, posibilita que la población LGBT construya estilos de vida heterogéneos con grados de libertad dispares" (p.146). De esta manera lo afirman Barret \& Pollack (2005), quienes señalan que la variable de clase social moldea la capacidad de las personas para expresar su orientación sexual. Sin embargo, para que esto sea representativo y promueva la equidad social, es importante el reconocimiento de los derechos y la protección LGBT (Sabharwal et al., 2018). En tal sentido, Jiménez et al. (2017) indican que "los procesos de discriminación contra la población LGBT generan que las relaciones sociales sean desiguales, incluso en los mercados laborales, afectando la calidad de vida de estos" (p. 233).

Para Pizer et al. (2012) "una gran proporción de personas LGBT experimenta discriminación en el lugar de trabajo a raíz de su orientación sexual y/o identidad de género" (p. 722); mientras que Bendick et al. (2010, como se citó en Sabharwal, 2014) señala que la construcción de un entorno inclusivo es una combinación de compromiso de los empleadores, respeto por diversas opiniones y perspectivas. Es así que los entornos de trabajo inclusivos buscan aprovechar la variedad de sus colaboradores, ofreciéndoles una plataforma donde los empleados son tratados como un activo versus un pasivo (Sabharwal, 2014).

Las organizaciones de empleadores están adoptando cada vez más posturas de apoyo hacia las personas de comunidades LGBT, que incluyen políticas de no discriminación de identidad de género (Pichler et al., 2017). En Estados Unidos, el gobierno federal ha desarrollado políticas progresivas de derechos civiles con respecto a leyes antidiscriminatorias en el lugar de trabajo para ciudadanos estadounidenses lesbianas, gays, bisexuales y transgénero (LGBT), para la contratación de este grupo en sus espacios de trabajo (Sabharwal et al., 2018).

Más de la mitad de los estados de Estados Unidos de América carecen de una legislación de discriminación laboral que incluya al grupo LGBT (Cech \& Pham, 2017); sin embargo, señalan que varios estados están buscando activamente dejar atrás esta situación, ya que esto limita el acceso a algunos beneficios. En otra investigación realizada en ese país se evidenció que el lugar de trabajo federal ha sido el ente que ha aceptado más informalmente a los LGBT que la mayoría de los empleadores durante décadas (Lewis \& Pitts, 
2015). En ese sentido, las organizaciones han considerado relevante y pieza clave para las empresas la planeación estratégica de una imagen corporativa (Duque \& Carbajal, 2015).

Por otro lado, el movimiento LGBT ha tenido presencia y participación en el arte cinematográfico estadounidense, ya que "el cine se ha caracterizado por proyectar una multiplicidad de discursos que permiten identificar a ciertos espectadores con argumentos determinados (Chaparro \& Estefan, 2011, p. 57). En otro estudio realizado, también en Estados Unidos, se evidenció que a medida que se modifican las políticas de empleo, como la introducción de beneficios para los cónyuges del mismo sexo, los LGBT pueden percibir un trato más igualitario en sus trabajos; además, por la rapidez con que el clima para los derechos LGBT parece estar cambiando, la investigación debe continuar monitoreando las tendencias en las actitudes hacia dichas comunidades (Sabharwal et al., 2018).

"El reconocimiento de derechos de la diversidad sexual y el derecho de la identidad de género es limitado en el continente latinoamericano" (López, 2018, pp. 171-175). Incluso, se señala que "en Colombia y México, la reacción conservadora hacia los derechos de este grupo LGBT ha impulsado estrategias de cabildeo, reforma y presión institucional debido a que no reconocen los derechos de las minorías sexuales" (López, 2018, p. 179). Un estudio realizado en México señala que el mundo laboral es el principal lugar donde la población LGBT ha sufrido discriminación (Boivin, 2014). Por otro lado, un estudio realizado en Chapinero, Colombia, señala que existe discriminación laboral hacia las personas LGBT "a raíz de prejuicios y estereotipos como la identidad sexual, lenguaje corporal y grado de masculinidad y/o feminidad del trabajador, lo cual desencadena en segmentación laboral por género y orientación sexual" (Jiménez et al., 2017, p. 245). En otros países del mundo, como Inglaterra, España o Argentina, se han visto cambios en cuanto a la situación sociolaboral, tanto de gays como lesbianas. Sin embargo, los actos de discriminación hacia este grupo siguen vigentes (Ortega et al., 2018).

En el caso del Perú, la realidad no dista mucho de la de otros países: no existen datos oficiales sobre la situación de las personas pertenecientes a estas comunidades ni tampoco políticas públicas en favor de ellas. De acuerdo con la "Encuesta para medir la opinión de la población peruana en relación a los Derechos Humanos", realizada en el año 2013 por el Ministerio de 
Justicia y Derechos Humanos con la colaboración de la Universidad ESAN, el 93\% de los encuestados indicó que las personas LGBT se encuentran más expuestas a la discriminación, así como al maltrato físico (88\%), al maltrato verbal (92\%), a las amenazas (84\%) y al chantaje (78\%). Asimismo, el $45 \%$ consideraba que las personas LGBT no deberían ser docentes en colegios y un $59 \%$ que no deberían tener derecho al matrimonio civil (Ministerio de Justicia y Derechos Humanos, 2013). En ese sentido, se planteó la siguiente pregunta de investigación: ¿cómo impacta la inclusión de miembros de comunidades LGBT en la imagen corporativa de tiendas retail en el Perú?

\subsection{Marco teórico}

\subsubsection{LGBT}

El término LGBT hace referencia a la comunidad de lesbianas (mujeres que tienen afinidad por otras mujeres homosexuales); gays (hombres que tienen afinidad por otros hombres homosexuales); bisexuales (personas orientadas erótico-afectivamente hacia ambos sexos); transgénero (personas que rompen las definiciones tradicionales de hombre y mujer respecto a su físico, actitudes y gestos), según la Fundación Colombia Diversa (2005, como se citó en Duque, 2008). Con relación a ello, Diamond (2003), Rosario et al. (2006) y Saewyc et al. (2004, como se citó en Russell \& Fish, 2016) señalan que el término LGBT es un acrónimo utilizado para referirse a las personas que seleccionan esas etiquetas de identidad sexual o de género y tiene un impacto personalmente significativo para ellos y las identidades sexuales y de género.

Desde un punto de vista sociológico, Fraser (1997, como se citó en Jiménez et al., 2017) señala que el grupo de lesbianas, gays, bisexuales y transgénero, conocido por el acrónimo LGBT, es considerado como una comunidad que no tiene el reconocimiento como sujetos sociales. Por otro lado, para Sabharwal et al. (2018), tanto las lesbianas, gays, bisexuales como los transgénero (LGBT), son un grupo de personas que han sido marginadas a través de la negación sistemática de beneficios legales. Además, durante décadas, han sido víctimas de discriminación, marginación, acoso y la negación de recursos (Cech \& Pham, 2017). Es así que las personas lesbianas, gays, bisexuales y transgénero (LGBT) experimentan violencia y discriminación (Swan et al., 2019). 
En tal sentido, las personas lesbianas, gays, bisexuales y transgénero (LGBT) tienen mayores probabilidades de autodeclarar depresión, ansiedad, ideación o intentos suicidas, consumo excesivo de sustancias, tener conductas sexuales de riesgo, por mencionar algunas (Juster et al., 2019).

\subsubsection{Inclusión de minorías}

Los grupos minoritarios son más vulnerables socialmente y, a raíz de dicha vulnerabilidad, cuentan con una nula participación en el proceso de toma de decisiones en los distintos grupos sociales existentes (Mantovani de Lima \& Bergamaschine, 2015). Por ello, (Meyer, 1995, como se citó en Barrientos \& Cárdenas, 2013) "las personas que forman parte de grupos minoritarios están expuestos a estresores sociales, tales como prejuicio, rechazo" (p.8), por lo cual "presentan niveles inferiores de bienestar, respecto a la población en general" (Riggle et al, 2009, como se citó en Barrientos, 2013, p. 8).

La inclusión busca maximizar la presencia, participación, e implica el reconocimiento y valoración de distintas culturas e identidades (Duk \& Murillo, 2016). Asimismo, la inclusión se enfoca en la eliminación de barreras para permitir un alto desempeño de todos (Miller, 1998; MorBarak \& Cherin, 1998; Roberson, 2006, como se citó en Sabharwal, 2014). En tal sentido, Ramchand \& Fox (2007, como se citó en Mayer et al., 2008) señalan que los individuos LGBT continúan encontrando barreras para la inclusión, al ser considerados minorías sexuales y de género. Se señala (Mayer et al., 2008) que algunas personas LGBT deciden renunciar a revelar su identidad sexual o de género para tener acceso a la atención médica. Por su parte, Gregory (2007), Tilcik (2011) y Weichselbaumer (2003, como se citó en Cech \& Pam, 2017) coinciden con Mayer et al. (2008) y señalan que dentro de las organizaciones en donde se incluyen empleados LGBT, estos últimos enfrentan políticas discriminatorias formales e informales, como los beneficios de salud.

En relación con ello, Lewis \& Pitts (2017, como se citó en Sabharwal et al., 2018) señalan que, en el campo laboral, los empleados LGBT tienen un nivel inferior de satisfacción respecto de aquellos no LGBT, lo cual tiene impacto, también, en sus evaluaciones de desempeño, compromiso con la diversidad, liderazgo de la agencia, entre otros. 


\subsubsection{Imagen corporativa}

"La imagen corporativa es la impresión general fabricada en las mentes del público acerca de una empresa" (Duque \& Carvajal, 2015, p. 117). Por otro lado, Gunalan \& Ceylan (2014) señalan que la imagen corporativa es una percepción actual que los públicos, tanto externos como internos, tienen sobre la organización en comparación con otras organizaciones. Por su parte, Gronroos (1988) \& Keller (1993, como se citó en Lai et al., 2009) coinciden con Gunalan \& Ceylan (2014) e indican que la imagen corporativa es la percepción que un individuo mantiene en su memoria sobre una organización.

Asimismo, se describe como la impresión general que se tiene sobre la organización y se genera en las mentes de las personas (Barich \& Kotler, 1991, como se citó en Huang \& Lien, 2012). Por su parte, Bromley (2001) y Balmer (2001, como se citó en Pérez \& Rodríguez del Bosque, 2014) indican que la imagen corporativa consiste en la representación mental global que un individuo tiene sobre la organización, la cual se constituye por una serie de asociaciones, ya sean de carácter afectivo como racional, que se manifiestan en la mente de cada ser. Además, Moodley et al. (2008, como se citó en Huang \& Lien, 2012) señalan que la imagen corporativa de una empresa es una extensión de su marca de servicio y, además, un indicador importante de confianza entre el cliente y la empresa.

"La imagen corporativa es el resultado de la integración en la mente de los públicos con los que la empresa se relaciona, de un conjunto de imágenes que la empresa proyecta hacia el exterior" (Villafañe, 1998, como se citó en Currás, 2010, p. 22). A su vez, la imagen corporativa se considera como algo interno a la compañía; es decir, se enfoca en la creencia que los miembros de la organización tienen respecto a cómo son percibidos como empresa por sus públicos externos (Dutton et al., 1994, como se citó en Bravo et al., 2011).

Por otro lado, Sánchez \& Pintado (2013, como se citó en Apolo et al., 2017) definen la imagen corporativa como la conformación de atributos que cada individuo forma en su mente sobre una empresa. 


\section{Metodología}

El término retail proviene de la lengua inglesa y se utiliza para denominar a la venta minorista. Sin embargo, se vincula con la comercialización de grandes volúmenes de productos a compradores minoristas o clientes finales ${ }^{1}$. En ese sentido, se conoce como empresas de retail a las cadenas de supermercados, a tiendas por departamentos, de artículos de ferreterías, cadenas farmacéuticas, etc. El propósito de esta investigación fue analizar el impacto que tiene la inclusión de personas LGBT en la imagen corporativa de un grupo de empresas de retail en el Perú, específicamente en aquellas con la venta de ropa; es decir, cadenas de tiendas por departamentos y aquellas cadenas de marcas internacionales presentes en el país.

Para ello, se aplicó un diseño de tipo fenomenológico, ya que este responde al objetivo del estudio. Fuster (2019) señala que este tipo de diseño fenomenológico "surge como respuesta al radicalismo de lo objetivable" y tiene como base de estudio las experiencias de vida a partir de la óptica del individuo (p.202). Teniendo en cuenta que el tema planteado ha sido abordado en el ámbito internacional, pero no tanto en el ámbito nacional, se ha considerado pertinente que el alcance de investigación sea exploratorio.

Se optó por un planteamiento metodológico mixto, ya que se adecuaba a las necesidades de la investigación, utilizando tanto el enfoque cualitativo como cuantitativo. El enfoque cualitativo, según Blasco \& Pérez (2007), "estudia la realidad de lo que ocurre en el contexto natural", sin que este sea alterado, es decir, tal y como ocurren, para así "sacar e interpretar los fenómenos de acuerdo con las personas implicadas" (p. 2). Por su parte, Monje (2011) coincide con lo dicho por Blasco \& Pérez (2007), señalando que dicho enfoque tiene como interés captar la realidad de las personas que están siendo estudiadas, a partir de la percepción que estos tienen de su propio contexto. El enfoque cuantitativo, según Monje (2011) se basa en la recolección de información a través de "empíricos medibles, además de analizar la información recolectada, con el objetivo de determinar el grado de significación de las relaciones entre las variables" (p. 13). Respecto al paradigma de investigación, es de carácter interpretativo, ya que, según 
Ricoy (2006), lo que busca este paradigma es plantear "diseños abiertos y emergentes a partir de la globalidad y contextualización” (p. 17), y, con ello, constituir una reflexión a partir de los hechos observables.

La muestra de estudio estuvo conformada por 12 personas entrevistadas y 501 personas encuestadas de 18 a 60 años, que han comprado en tiendas retail ubicadas dentro de centros comerciales, tales como Saga Falabella, Ripley, Oeschle, Paris, Zara, H\&M, Forever 21 y similares. Cabe destacar que el trabajo de campo, tanto cualitativo como cuantitativo, fue culminado días antes de la declaración de la emergencia sanitaria y de la cuarentena en el Perú.

Las técnicas de recolección de datos fueron entrevistas en profundidad y encuestas. Según (Monje, 2011), el objetivo de la primera técnica consiste en la formulación de interrogantes por parte del investigador y la obtención de respuestas por parte del sujeto. En el caso de las encuestas, esta técnica sirve para estudiar un hecho determinado a través de la "identificación de contenidos y la preparación de un formulario" (p. 136), para lo cual, el investigador elabora una guía general de discusión.

El instrumento en las entrevistas en profundidad fue semiestructurado e incluyó preguntas abiertas para que el grupo de estudio tenga la posibilidad de profundizar en sus respuestas, según lo crea conveniente. Se utilizó el método de muestreo no probabilístico para la selección de los participantes y la técnica de cuotas, ya que se segmentó a la población de estudio en grupos por edades para identificar si existían diferencias o similitudes en las posturas frente a la inclusión de colaboradores LGBT en tiendas retail. Para ello, en las entrevistas en profundidad, se dividió la muestra en dos grupos de participantes: de 21 a 35 años y de 40 a 60 años. El primer grupo estuvo conformado por 2 mujeres y 4 hombres; mientras que el segundo grupo estuvo conformado por 4 mujeres y 2 hombres.

El objetivo de las entrevistas en profundidad fue hacer un primer levantamiento de información para conocer las posturas de los participantes y, a partir de la información obtenida, procesarla para elaborar el formulario de la encuesta. La encuesta buscó explorar si los hallazgos obtenidos con las entrevistas tenían sustento o no. Para ello, se diseñaron preguntas abiertas, cerradas y demográficas. Las preguntas abiertas buscaron que los participantes expresen sus ideas de manera libre; las cerradas, para confirmar si existe o no una 
tendencia en la información recolectada previamente de las entrevistas; y las demográficas para conocer y comparar si existe alguna diferencia o similitud en las respuestas de los encuestados con la muestra de entrevistados previamente.

\section{Resultados}

\subsection{Identificación de personas LGBT en tiendas retail}

E1 70.7\% de los participantes aseguró haber identificado a por lo menos una persona de la comunidad LGBT en algunas de las tiendas a las que asistió e indicó su posición frente al hecho de que personas de la comunidad LGBT trabajen en este tipo de tiendas. De ellas, el $87.6 \%$ señaló que no le afectaba y le era indiferente encontrarse con personas de la comunidad como colaboradores en dichas tiendas. Sin embargo, consideraron que el tener personal LGBT influye para considerar a las marcas como inclusivas.

\subsection{Experiencia/atención de personas LGBT en tiendas retail}

Respecto a las experiencias de relacionamiento con personas LGBT en tiendas retail, los entrevistados señalaron que existe una tendencia de mejora en el trato que brindan las personas de la comunidad al público, pues consideran que son más amables y tienen mayor predisposición para atender y asesorar a los consumidores, en lo que a vestimenta respecta. Por ejemplo, se señaló que cuando han estado en un establecimiento comercial, "trabajadores LGBT se les acercaron para asesorarlos y ayudarlos, ya que estaban en búsqueda de una prenda de vestir". También consideraron que las personas de la comunidad LGBT "tienen más ganas de atender que otros colaboradores". Otras de las características que señalaron es que no piensan que la atención de personas de la comunidad LGBT sea similar a la de aquellos que no pertenecen a ella, ya que, según ellos, "tienen una calidad de atención mejor, muestran interés, hacen sentir al cliente importante y sienten que el acompañamiento desde que entran al establecimiento comercial es bueno".

Los entrevistados consideraron que, en cuanto a atención, todo depende de la personalidad de la persona y no piensan que su orientación sexual influya 
en esto. Asimismo, señalaron que, debido a su personalidad, tienen un lado más cordial y "debería incluírseles porque son personas con una personalidad extrovertida. Sin embargo, si bien consideraron que son más atentos en su manera de tratar y más responsables, no piensan que "todos sean así'. Los entrevistados también aseguraron percibir que "la atención que brindan las personas de la comunidad es más personalizada, pues están más pendientes a las necesidades de los clientes, cuando estos lo requieren".

No obstante, hubo entrevistados que indicaron que han sido testigos de situaciones en las que personas no han querido ser atendidos por colaboradores LGBT. Esto, según señalan, se basa en prejuicios y estereotipos preestablecidos de antaño, en donde se especulaba que las personas LGBT "tenían enfermedades como el sida" y "se les solía asociar a las enfermedades venéreas como herpes, papiloma, VIH, incluso psicológicas, además que establecer contacto con ellos los exponía a contagios". Además, de algunos entrevistados se desprende que "se ha creado esa desconfianza que son raros, pueden tener enfermedades". Se señaló que el establecer vínculos con personas de la comunidad los podría clasificar como "raritos".

De los 501 participantes encuestados, el 77\% indicó que no encuentran diferencias en la atención o trato que reciben de parte de personas de la comunidad LGBT. Los encuestados consideraron que la orientación sexual de una persona no interfiere en el tipo de atención que las personas LGBT entregan. Asimismo, agregaron que deben basarse en las capacidades y desempeño profesional de las personas para atender de las personas. Otra característica que comentaron fue que consideran que no deberían existir estereotipos sobre las personas LGBT, ya que el trato no depende de su orientación sexual, sino de su personalidad. También indicaron que no han evidenciado diferencias en el trato, ya que consideraron que todos los trabajadores, en general, deben respetar los protocolos de atención y cumplir las funciones por igual para que el cliente se sienta satisfecho. También señalaron que no encontraban diferencias en el trato que dan las personas LGBT, mientras sean respetuosos y tengan modales. Por otro lado, hubo quienes señalaron que no consideran que habilidades blandas como servicio al cliente y relacionamiento dependan de la orientación sexual de las personas. Además, indicaron que tanto las personas LGBT como las personas que no pertenecen a dicha comunidad tienen derecho a trabajar y no deberían ser discriminados laboralmente por ser LGBT. 
El 23\% de los encuestados indicó que sí existían diferencias en la atención que brindaban las personas LGBT respecto a quienes no pertenecen a la comunidad. Los encuestados señalaron que características de la personalidad están relacionadas con el desempeño laboral de las personas LGBT, justificando que son personas "más sociables, carismáticas, atentas, cordiales, alegres, entusiastas, perfeccionistas, detallistas" y que esto influía en el trato que les dan a los clientes. También consideraron que encuentran diferencias en la atención, ya que las personas LGBT suelen orientarlos "mejor", porque saben más de moda y estilo. Otra de las características que consideraron como un diferenciador fue que los otros trabajadores LGBT tienen más predisposición para ayudar al cliente durante el proceso de compra y el ser más serviciales.

\subsection{Inclusión de colaboradores LGBT en tiendas retail}

En cuanto a la inclusión de personas LGBT en el ámbito laboral, los entrevistados señalaron que debería normalizarse la contratación de estas personas de la comunidad, pues "no tendría por qué importar su opción sexual", sino "enfocarse en sus capacidades para desempeñar el puesto de trabajo al que se postula". Además, indicaron que debería ser una acción "natural" contratar a personas LGBT y no un obstáculo para su contratación. Agregaron que, como cualquier persona, quienes pertenecen a la comunidad tienen derecho al trabajo. También señalaron "no tener nada en contra de los gays y que su inclusión en el campo laboral les parecería bien”.

Por otro lado, los entrevistados desestimaron la premisa de desigualdad según orientación sexual, pues consideran que "las empresas deben buscar igualdad entre sus colaboradores". Sin embargo, hubo quienes agregaron que están expuestos a posibles actos de discriminación por su orientación sexual, ya sea por parte del público externo como de sus compañeros de trabajo.

También algunos entrevistados señalaron que la apariencia física de una persona de la comunidad podría generar diversas reacciones en el público a este tipo de tiendas. Es decir, señalaron que podría generar asombro o posturas negativas al ver a un hombre maquillado, porque "la gente lo puede ver raro". Otras expresiones fueron: "si vas a ir a un establecimiento maquillado y vestido de hombre, no es discriminación, pero como que te sorprendes"; "mientras lleven por dentro su opción, creo que todo bien"; "si 
lo ves a primera vista no te das cuenta, pero cuando te atiende quizás sí, he conocido algunos que paran con el cabello... hacen sus gestos"; "socialmente una persona LGBT es discriminada por su forma de vestir, a veces en el trabajo no entienden las opciones sexuales, y genera problemas de aceptación que se traducen en varios ambientes".

En términos generales, los encuestados coincidieron con lo expuesto por los entrevistados: les parece bien que las empresas contraten a personas de la comunidad, ya que eso los hace más "inclusivos", ayuda a disminuir las brechas de desigualdad y elimina prejuicios. Además, señalaron que el pertenecer al grupo LGBT no tiene relación directa con su rendimiento laboral, ya que no consideraron que sea un factor que determine las capacidades de las personas para desempeñarse en un puesto de trabajo. Asimismo, señalaron que tanto las personas LGBT como las no LGBT merecen las mismas oportunidades sin diferenciación. Sin embargo, recalcaron que no les parece mal la inclusión de personas de la comunidad "mientras cumpla con las normas de comportamiento y trabajo, y no involucren sus ideologías en el trato y atención". También señalaron que es normal contratar a personas de la comunidad, "porque ya no es un tabú y se está venciendo la homofobia". Por su parte, hubo quienes señalaron que en muchas empresas existen personas de la comunidad; sin embargo, por temor a ser rechazados "visten de corbata". Hubo quienes señalaron que debía incluirse a personas de la comunidad LGBT, ya que consideraron que son personas más atentas, eficientes y ordenadas.

Por otro lado, algunos encuestados señalaron que les era indiferente la inclusión de personas LGBT en tiendas retail, ya que no les afecta "mientras se dediquen a trabajar". Además, recalcaron que son personas como todas y deberían ser considerados en la contratación siempre y cuando cumpla con los requisitos del puesto al que postula. No obstante, hubo algunos que señalaron que no consideran que "las personas LGBT deban estar en puesto de atención al cliente, sino solo en fábricas, ya que alteran el orden natural de la especie humana".

\subsection{Cuota de personas LGBT}

En cuanto a la exigencia a las empresas de una cuota mínima de contratación de personas LGBT, hubo discrepancias, pues hubo entrevistados que consideraron que sí debería establecerse a las empresas un número determinado 
de personas LGBT entre todos sus colaboradores y hubo quienes indicaban que no debería delimitarse la cuota como tal, ya que "no debería obligarse a las empresas a contratar a personas LGBT solo por ser de la comunidad, sino por las capacidades que tienen". Así como señalaron estar de acuerdo con la inclusión, también se mencionó que "el debate que se ha generado parece tonto porque son personas y no habría por qué discriminarlos. Es una tontería que la gente a veces no quiera ser atendido por ellos".

Los participantes encuestados, al igual que los entrevistados, optaron por dos posturas con relación a una cuota mínima de personas LGBT en empresas. Por un lado, el $51.9 \%$ de encuestados señaló que no considera que las empresas deban tener una cuota mínima de trabajadores LGBT, ya que la segmentación de trabajadores por orientación sexual demostraría que existen diferencias en ellos, y no debería ser así, pues reafirmaría el concepto de discriminación. Además, consideraron que al establecer una cuota mínima se estaría contradiciendo con el término de inclusión, ya que son personas como cualquiera y deben ser tratados por igual.

Con relación a ello, señalaron no estar de acuerdo con una cuota mínima, ya que no debería existir favoritismos: "las personas deben ganarse su puesto sin importar su orientación sexual y basarse en sus capacidades". Además, señalaron que todas las personas deben tener las mismas oportunidades de aplicar a un trabajo. Hubo quienes indicaron que no se les debería obligar a las empresas a incluir a colaboradores LGBT, que no debería ser una norma, sino que las empresas deberían hacerlo solo si lo desean. Además, señalaron que la existencia de una cuota implicaría condicionar el proceso de contratación; es decir, las personas LGBT no poseen ningún limitante en sus capacidades, ya que su "rasgo diferenciador" es únicamente la orientación sexual, por lo que deberían ser incluidos en los procesos regulares. Consideraron irrelevante el tema de la cuota mínima, ya que "cuando se postula a un trabajo no te preguntan si eres gay, lesbiana, transexual”.

Asimismo, señalaron que la orientación sexual de una persona no debe ser un requisito para obtener un puesto de trabajo, ya que "el pertenecer a la comunidad no te hace ser discapacitado y no se debería favorecer a un grupo en especial, pues todas las personas tienen necesidades y están en busca de oportunidades laborales". Por otro lado, hubo quienes no están de acuerdo con la cuota mínima de personas LGBT; sin embargo, señalaron que depende 
de lo procesos internos de cada empresa y los perfiles que estas soliciten. Asimismo, indicaron que la orientación sexual no es algo tan visible y la existencia de una cuota requeriría que una persona revele su orientación sexual o su identidad, lo cual no sería algo justo para los trabajadores, ya que se expondrían a la discriminación. Con relación a ello, señalaron que hay rubros en donde las personas LGBT sufrirían si se les obliga a las empresas a contratarlos.

Por otro lado, el $48.1 \%$ de los encuestados sí consideró que las empresas deben tener una cuota mínima de trabajadores LGBT, porque hay muchas personas que pertenecen a dicha comunidad y son discriminados por su orientación y, al haber una cuota, se les daría más oportunidades de trabajo. Además, señalaron que esto ayudaría a que las personas LGBT pierdan el miedo de no conseguir empleo por decir que pertenecen a dicha comunidad. También, que esto puede ayudar a cambiar la mentalidad de las personas y así entender que las personas LGBT merecen las mismas oportunidades que los demás. "Lamentablemente, aún hay empresas discriminadoras y personas de la comunidad LGBT entran como personas vulnerables; por ello, consideraron que para que haya un balance, se debería de pedir una cuota, para que todos tengan la posibilidad de trabajar sin ser discriminados por su orientación sexual, al menos hasta que se interiorice la idea de que no hay razón para discriminar".

Los encuestados indicaron que las personas LGBT "muchas veces son discriminadas y tienen más dificultades para encontrar un puesto de trabajo, alegando que no cumplen con los requisitos para el puesto, disfrazando de esa manera la discriminación y cerrándoles oportunidades en el mundo laboral". Hubo encuestados que señalaron que esto ayudaría a promover la inclusión, diversidad y reducción de desigualdad; además de fomentar en la sociedad la costumbre de convivir con ellos. Asimismo, señalaron que la cuota es una forma de asegurarse que no se descarte a personas LGBT en el proceso de selección por homofobia y garantiza que no sean discriminados por temas ajenos a las competencias de su puesto. Por otro lado, indicaron que sería como un apoyo y visibilidad positiva a esta comunidad, ya que fomenta la costumbre de convivir con ellos, teniendo en cuenta que es una minoría maltratada en esta sociedad. La implementación de una cuota LGBT en las empresas influiría a otras empresas a incluir a trabajadores LGBT en su planilla. 


\subsection{Influencia en la asistencia de tiendas retail}

El 93.8\% de los participantes encuestados aseguraron que la presencia de personas LGBT no influía en su asistencia a este tipo de tiendas, ya que indicaron que concentran su atención en los productos que comprarán y no en la sexualidad de las personas que los atienden. Además, señalaron que el principal atractivo para asistir a este tipo de tiendas se debe al factor económico, pues asisten cuando hay descuentos y promociones. También señalaron no tener prejuicios en relación con las personas de la comunidad LGBT. Asimismo, indicaron que cuando asisten a este tipo de tiendas buscan un servicio de atención de calidad sin importar su orientación sexual.

El 6.2\% de los encuestados señaló que la presencia de colaboradores LGBT sí influía en su asistencia a este tipo de tiendas, ya que indicaban que el tener colaboradores LGBT en este tipo de tiendas sirve para visibilizarlos, además de resaltar que consideran que su atención es más personalizada. Sin embargo, hubo quienes indicaron que no asistirían a este tipo de tiendas con niños: "no llevaría a mis hermanitos menores", "sí influye porque voy con mis hijos".

\subsection{Impacto en la imagen corporativa}

Respecto al impacto que podría tener la imagen de las empresas al incluir personal LGBT, los entrevistados señalaron que no consideran que afectaría de forma negativa la imagen, porque "los tiempos han cambiado". Consideraron que no creen que impacte mucho, porque ya se han hecho la idea de que "van a verlos en varios campos de sus vidas". Además, "una empresa que los contrata es considerada más inclusiva, que apoya la diversidad, más libertad de aceptación con personas LGBT". Otra declaración fue "siento que la mayoría de las personas que me han atendido son personas jóvenes gays". También señalaron que las empresas que incluyen personal LGBT buscan igualdad.

Los entrevistados comentaron que las personas de la comunidad aportan aspectos positivos a la empresa, ya que son personas "respetuosas, positivas, luchan por un trabajo y quieren superarse". Los entrevistados descartaron que la presencia de personas LGBT influya en el orden que puede tener un establecimiento, señalando que esto es relativo y depende de los jefes que 
cada uno tiene, "porque todo tiene que salir como el jefe dice", "lo he visto igual, no he notado diferencias, siempre está ordenado".

También señalaron que las empresas van a enfrentar desafíos al incluir trabajadores LGBT, pues habrá personas que no lo aceptarán y otros que sí. "El mayor desafío va a ser romper esta barrera: que son iguales como cualquier otra persona". Asimismo, consideraron que "las personas que están en contra de ellos no creen que cambien su punto de vista, ya que antiguamente lo correcto era marginarnos; sin embargo, ahora no, y que todo es cuestión de tiempo".

El $62.5 \%$ de los encuestados no consideraron que la presencia de colaboradores LGBT afecte la imagen de una empresa, el $32.3 \%$ señaló que afecta positivamente y el $5.2 \%$ indicó que afecta de forma negativa. En primer lugar, quienes señalaron que la imagen de una empresa no se ve afectada por la presencia de colaboradores LGBT argumentan que lo que se valora es la calidad de atención en el trabajo. Asimismo, hubo quienes señalaron que la presencia de personas de la comunidad no aporta, solo "se intenta congraciar con ese sector".

\section{Figura 1}

Percepciones ante la presencia de personas LGBT en tiendas retail

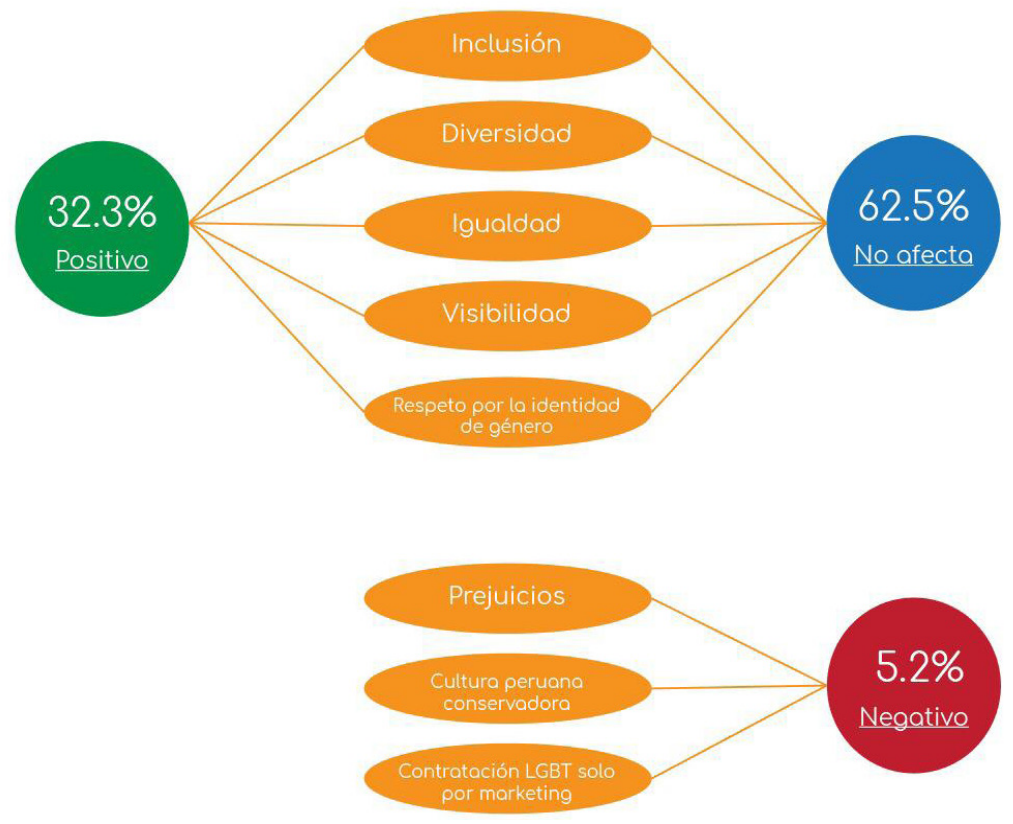

Nota: Elaboración propia. 
Entre algunas respuestas abiertas, se destacan aquellas que expresaron que no debería existir diferenciación de personas por ningún aspecto, por lo que no se debería pensar si la presencia de personas LGBT aporta de forma positiva o negativa en la empresa. Con relación a ello, indicaron que no se debería contratar a personas LGBT solo para beneficiar a la marca. Los encuestados indicaron que son las personas quienes deben cambiar su forma de pensar y aprender a aceptar la presencia de personas LGBT. "Mientras hagan bien su trabajo y la atención sea buena, no afecta la imagen”.

A pesar de que el $62.5 \%$ de los encuestados consideró que la presencia de colaboradores LGBT no afecta la imagen de una empresa, los participantes argumentaron aspectos positivos que aporta la presencia de estas personas en las empresas, como la inclusión, igualdad, orientación sobre tendencia en moda, integración y respeto por la identidad de género. Además, señalaron que la empresa refleja una imagen de que no es discriminatoria y que cumple con la normativa de no discriminar al contratar. Por otro lado, los encuestados señalaron que el público al que se dirigen las empresas es un público joven, por lo que tienen "la mente más abierta" y comprenden mejor el sentido de igualdad. Sin embargo, hubo quienes indicaron que la imagen de la empresa no se ve afectada "mientras no se mezcle lo laboral con lo personal" y consideran que "mientras no sean escandalosos, no dan mala imagen a la empresa"

A su vez, el 32.3\% de los encuestados señaló que la presencia de trabajadores LGBT afecta la imagen de una empresa de forma positiva. Ello porque consideran que las hace ver como empresas que apoyan la diversidad, igualdad, las personas se acostumbran a los nuevos estereotipos. Además, indicaron que la presencia de colaboradores LGBT mejora la imagen de marca y valores de la empresa. Los encuestados indicaron que la contratación de personas LGBT expresa que la empresa respeta los derechos de las personas y da oportunidades a quienes lo necesitan. Asimismo, consideraron que la presencia de colaboradores LGBT da a entender al público que la empresa no tiene ningún tipo de estereotipo ni discriminación hacia este grupo de personas. Además de proyectar que es una empresa más inclusiva y open mind (mente abierta); es decir, señalan que la empresa demuestra que es abierta hacia otras opciones. Señalan que "hoy en día, las personas que no sean heterosexuales son muy discriminadas y las empresas que las incluyen proyectan una imagen de que no existe discriminación allí”. 
E1 5.2\% indicó que la presencia de colaboradores LGBT aportaba una imagen negativa en las empresas, ya que los peruanos son muy "conservadores" y creen que parte de ser una persona "correcta" se encuentra rechazar a los homosexuales, porque es una ideología que va en contra de su "religión". Con relación a ello, señalan que la sociedad es un factor importante y las familias en Perú tienden a ser conservadoras y, por ende, tienen estereotipos preestablecidos respecto a personas de la comunidad. Por otro lado, indicaron que en caso todo el personal tuviera "esa opción de ser LGBT, podría confundir a menores de edad". También se resaltó el comportamiento y forma de vestir como aspectos negativos. Consideraron que, en la sección de moda, las personas con "mejor ojo" son las mujeres y los homosexuales y es por eso que, en la industria de la moda, la mayor cantidad de diseñadores, modistas, son mujeres y homosexuales. Resaltaron que muchas veces las empresas contratan a personas LGBT solo por marketing y para beneficiar la imagen de la empresa.

\section{Discusión y conclusiones}

Tomando en consideración los resultados de la investigación, se comprobó lo expuesto por Jiménez et al. (2017) respecto a que "existe discriminación laboral hacia las personas LGBT a raíz de prejuicios y estereotipos como la identidad sexual, lenguaje corporal y grado de masculinidad y/o feminidad del trabajador" (p. 245). Esta afirmación se relaciona con los resultados obtenidos, ya que la muestra seleccionada confirmó que estas ideas se encuentran en la sociedad y son usadas para juzgar de forma negativa a las personas que pertenecen a dicho grupo. Sin embargo, aseguraron que esto tenía origen en la cultura conservadora en la que se basa la mayoría de las personas de Perú, en donde lo que rompe la regla de lo establecido como normal, se considera diferente y se suele marginar.

Por otro lado, según Jiménez et al. (2017) "a raíz de dichos prejuicios en contra de las personas LGBT se desencadena una segmentación laboral por género y orientación sexual" (p.245). Esto se confirma parcialmente con lo señalado por un grupo de los encuestados, ya que aseguraron que suelen haber separaciones de perfil por características que creen estar relacionadas a personas de la comunidad en cuanto a habilidades blandas, como, por ejemplo, una mayor predisposición para atender al cliente. 
Sin embargo, otro grupo aseguró que esta última premisa no es cierta, ya que se identificó que la orientación sexual de los trabajadores no tiene influencia en la calidad de atención. Asimismo, no es pertinente hacer distinción entre los trabajadores, pues se estaría aceptando que son diferentes, ya que hacer segmentación entre personas LGBT y no LGBT en las empresas reafirma el concepto de discriminación. A raíz de ello, no consideraron que deba establecerse en las empresas retail el concepto de una cuota mínima que incluya a personas de la comunidad entre su planilla de trabajadores porque, al igual que aquellos que no son parte de la comunidad, están regidos por lineamientos internos de las empresas y deben respetarlos sin importar la orientación sexual que tengan. Por otro lado, pese a que casi la mayoría de los participantes coincidió con lo señalado anteriormente, se identificó que se asociaron variables relacionadas con la moda, por la que sí debía hacerse una segmentación de estas personas, ya que se considera que tienen el conocimiento para asesorarlos en este tema.

También se identificó lo expuesto por Pizer et al. (2012), acerca de que "la población LGBT experimenta discriminación en el lugar de trabajo a raíz de su orientación sexual y/o identidad de género" (p.722). Sin embargo, se contrasta con esta investigación, ya que, si bien los participantes aseguraron que este grupo de personas a lo largo de los años ha sido discriminado por su condición, no se considera que esto deba ser un factor que limite su acceso en el mundo laboral; pues señalaron que debe existir una normalización de este grupo entre la sociedad y así eliminar la brecha de desigualdad, además de tener en cuenta las capacidades, habilidades y competencias que se requieran para un puesto de trabajo, sin importar si es LGBT o no. Se identificó que la presencia de colaboradores LGBT en tiendas retail no incide en la asistencia de personas a este tipo de tiendas, ya que priorizan otros factores como atención, precio y características del producto, antes que la orientación sexual de quien los atiende.

Se evidenció que no se cumple lo propuesto por Barret \& Pollack (2005) en cuanto a la "falta de aceptación en los estilos de vida de las personas LGBT" (p.438), pues los participantes aseveraron no importarles la vida íntima $\mathrm{u}$ orientación sexual de los trabajadores, ya que su asistencia a tiendas retail se enfoca en el proceso de compra de un producto y no en el personal que los atiende. Si bien consideraron importante el trato que reciben en tiendas, desestimaron una relación en cuanto a estilos de vida de las personas y su 
desempeño laboral, ya que no consideran que sea un factor que determine las capacidades de las personas para desempeñarse en un puesto de trabajo.

Por otro lado, Bendick et al. (2010, como se citó en Sabharwal, 2014) señalan que la construcción de un entorno inclusivo es una combinación de compromiso de los empleadores, respeto por diversas opiniones y perspectivas. Ello se confirma con los resultados obtenidos, ya que insisten que los empleadores no deben interferir en la vida íntima de sus trabajadores. Asimismo, lo expuesto por Duk \& Murillo (2016) en torno a la inclusión, señala que esta busca maximizar la presencia, participación e implica el reconocimiento y valoración de distintas culturas e identidades; lo cual sí se cumplió, pues la mayoría de la muestra expresó que un trato igualitario promueve la inclusión en las empresas y reduce los estereotipos asociados a este grupo.

Tanto los entrevistados como los encuestados no consideraron que la inclusión de colaboradores LGBT afecte la imagen de las organizaciones. Según Sánchez \& Pintado (2013, como se citó en Apolo et al., 2017), "la imagen corporativa es la conformación de atributos que cada individuo forma en su mente sobre una empresa" (p.532). El público de la investigación señaló que la imagen de las empresas no se ve afectada tras la inclusión de colaboradores LGBT. En sus argumentos lo asocian a aspectos positivos para las organizaciones, ya que estas demuestran que promueven la diversidad y la reducción de desigualdad en el entorno laboral. Además, asociaron que las empresas retail se dirigen en su mayoría a un público juvenil, por lo que existe una tendencia en estas empresas a ser inclusivas, diversas y promover la igualdad, ya que están abiertas al cambio.

Sobre la base de esta investigación, se concluye que existe una percepción a favor de la inclusión de población LGBT en tiendas retail en el Perú y que el actuar de las empresas en ese sentido impacta de manera positiva en su imagen corporativa; sobre todo en la población menor a los 40 años que no se encuentra influenciada por prejuicios o estereotipos. Dado que en el país no existen políticas públicas en favor de las comunidades LGBT, las acciones de las empresas que promueven la inclusión tienen un impacto positivo desde el punto de vista comunicacional, pues genera valor en aquellos activos intangibles, tales como el clima organizacional y la reputación. 


\section{Fuentes consultadas}

Apolo, D., Báez, V., Pauker, L., \& Pasquel, G. (2017). Corporate Communication Management: considerations for its study and practice. Revista Latina de Comunicación Social, 72, 521-539. http://www.revistalatinacs.org/072paper/1177/27en.html

Barret, D., \& Pollack, L. (2005). Whose Gay Community? Social Class, Sexual Self-Expression, and Gay Community Involvement. The Sociological Quarterly, 46(3), 437-456. https://doi.org/10.1111/ j.1533-8525.2005.00021.x

Barrientos, J., \& Cárdenas, M. (2013). Homofobia y Calidad de Vida de Gay y Lesbianas: Una Mirada Psicosocial. Psykhe, 22(1), 3-14. http://dx.doi.org/10.7764/psykhe.22.1.553

Blasco, J., \& Pérez, J. (2007). Metodologías de investigación en las ciencias de la actividad física y el deporte: ampliando horizontes. Editorial Club Universitario. https://rua.ua.es/dspace/ bitstream/10045/12270/1/blasco.pdf

Bravo, R., Matute, J., \& Pina, J. (2011). Efectos de la imagen corporativa en el comportamiento del consumidor. Un estudio aplicado a la banca comercial. Revista Innovar, 21(40), 35-51. https://www. redalyc.org/pdf/818/81822453004.pdf

Boivin, R. (2014). “Se podrían evitar muchas muertas". Discriminación, estigma y violencia contra minorías sexuales en México. Sexualidad, salud y sociedad - Revista Latinoamericana, (16), 86120. https://www.redalyc.org/pdf/2933/293330166006.pdf

Cech, E., \& Pham, M. (2017). Queer in STEM Organizations: Workplace Disadvantages for LGBT Employees in STEM Related Federal Agencies. Social sciences, 6(1), 12. https://doi.org/10.3390/ socsci6010012 
Chaparro, N., \& Estefan, S. (2011). Imágenes de la diversidad. El movimiento de liberación LGTB tras el velo del cine. Culturales, 7(14), 57-86. http://www.scielo.org.mx/scielo.php?script=sci_arttext\&pid=S1870$11912011000200004 \&$ lang=es

Currás, R. (2010). Identidad e imagen corporativas: revisión conceptual e interrelación. Teoría y Praxis, (7), 9-34. http://www.teoriaypraxis. uqroo.mx/doctos/Numero7/Curras.pdf

Duque, C. (2008). Aproximación teórica al debate por el reconocimiento político, jurídico y social del sector LGTBIQ. Revista CS, (2), 181207. https://www.redalyc.org/pdf/4763/476348366005.pdf

Duque, E., \& Carvajal, L. (2015). La identidad organizacional y su influencia en la imagen: una reflexión teórica. Suma de negocios, 6(13), 114-223. https://www.researchgate.net/ publication/283195180

Duk, C., \& Murillo, F. (2016). La Inclusión como Dilema. Revista latinoamericana de educación inclusiva, 10(1), 11-14. http://dx.doi. org/10.4067/S0718-73782016000100001

Fuster, D. (2019). Investigación cualitativa: Método fenomenológico hermenéutico. Propósitos y Representaciones, 7(1), 201-229. http:// dx.doi.org/10.20511/pyr2019.v7n1.267

Gunalan, M., \& Ceylan, A. (2014). The mediator role of organizational image on the relationship between jealousy and turnover intention: A study on health workers. Balikesir University The Journal of Social Institute, 17(31), 133-156. https://www.researchgate.net/ publication/274509977

Huang, C., \& Lien, H. (2012). An empirical analysis of the influences of corporate social responsibility on organizational performance of Taiwan's construction industry: Using corporate image as a mediator. Construction Management and Economics, 30(4), 263-275. https:// www.researchgate.net/publication/241715603 
Jiménez, J., Cardona, M., \& Sánchez, M. (2017). Discriminación y exclusión laboral en la comunidad LGBT: un estudio de caso en la localidad de chapinero, Bogotá Colombia. Papeles de población, 23(93), 231-267. https://rppoblacion.uaemex.mx/ article/view/9112/7654

Juster, R., De Torre, M., Kerr, P., Kheloui, S., Rossi, M., \& Bourdon, O. (2019). Sex Differences and Gender Diversity in Stress Responses and Allostatic Load Among Workers and LGBT People. Current Psychiatry Reports, 21(11). https://link. springer.com/content/pdf/10.1007/s11920-019-1104-2.pdf

Lai, F., Griffin, M., \& Babin, B. (2009). How quality, value, image, and satisfaction create loyalty at a Chinese telecom. Journal of Business Research, 62(10), 980-986. https://doi.org/10.1016/j. jbusres.2008.10.015

Lewis, G., \& Pitts, D. (2015). LGBT-Heterosexual Differences in Perceptions of Fair Treatment in the Federal Service. The American Review of Public Administration, 47(5), 574-587. https://www.researchgate.net/publication/282355014

López, J. (2018). Movilización y contramovilización frente a los derechos LGBTI. Respuestas conservadoras al reconocimiento de los derechos humanos. Estudios sociológicos, 36(106), 161187. https://doi.org/10.24201/es.2018v36n106.1576

Mantovani de Lima, R., \& Bergamaschine Mata Diz, J. (2015). El derecho de las minorías y los conflictos armados: la creación y puesta en marcha del Tribunal Especial para el Líbano. Anuario Mexicano de Derecho Internacional, (15), 571-610. https:/www.redalyc.org/ pdf/4027/402741202015.pdf

Mayer, K., Bradford, J., Makadon, H., Stall, R., Goldhammer, H., \& Landers, S. (2008). Sexual and gender minority health: what we know and what needs to be done. American Journal of Public Health, 98(6), 989-995. https://ajph.aphapublications.org/ doi/10.2105/AJPH.2007.127811 
Meccia, E. (2011). La sociedad de los espejos rotos. Apuntes para una sociología de la gaycidad. Sexualidad, Salud y Sociedad Revista Latinoamericana, (8), 131-148. https://www.redalyc.org/ pdf/2933/293322074006.pdf

Mejía, J., \& Almanza, M. (2010). Comunidad LGBT: Historia y reconocimientos jurídicos. Revista Jurídica, 15(17), 78-110. http://revistas.unisimon.edu.co/index.php/justicia/article/ view/618/606

Ministerio de Justicia y Derechos Humanos del Perú (2013). Encuesta para medir la opinión de la población peruana en relación con los derechos humanos. https://www.mimp.gob.pe/webs/mimp/ sispod/pdf/263.pdf

Monje, C. (2011). Metodología de la investigación cuantitativa y cualitativa. Guía didáctica. Universidad Surcolombiana. https:// www.uv.mx/rmipe/files/2017/02/Guia-didactica-metodologia-dela-investigacion.pdf

Ortega, J. (mayo, 2018). Heterosexismo internalizado en enfermeros $y$ enfermeras gays y lesbianas de la Ciudad de Buenos Aires [ponencia]. Latin American Studies in a Globalized World (LASA), Barcelona, España. https://www.researchgate.net/ publication/324824846

Pérez, A., \& Rodríguez del Bosque, I. (2014). Identidad, imagen y reputación de la empresa: integración de propuestas teóricas para una gestión exitosa. Cuadernos de Gestión, 14(1), 97-126. https:// www.redalyc.org/pdf/2743/274330593005.pdf

Pichler, S., Ruggs, E., \& Trau, R. (2017). Worker outcomes of LGBTsupportive policies: a cross-level model. Equality, Diversity and Inclusion: An International Journal, 36(1), 17-32. https://www. researchgate.net/publication/313661132

Pizer, J., Sears, B., Mallory, C., \& Hunter, N. (2012). Evidence of Persistent and Pervasive Workplace Discrimination Against LGBT People: 
The Need for Federal Legislation Prohibiting Discrimination and Providing for Equal Employment Benefits. Loyola of Los Angeles Law Review, 45(3). https://digitalcommons.lmu.edu/llr/vol45/iss3/3/

Ricoy, C. (2006). Contribución sobre los paradigmas de investigación. Revista do Centro de Educação, 31(1), 11-22. https://www.redalyc. org/pdf/1171/117117257002.pdf

Russell, S., \& Fish J. (2016). Mental Health in Lesbian, Gay, Bisexual, and Transgender (LGBT) Youth. Annual Review of Clinical Psychology, 12(1), 465-487. https://doi.org/10.1146/annurevclinpsy-021815-093153

Sabharwal, M. (2014). Is Diversity Management Sufficient? Organizational Inclusion to Further Performance. Public Personnel Management, 43(2), 197-217. http://dx.doi.org/10.1177/0091026014522202

Sabharwal, M., Levine, H., D’Agostino, M., \& Nguyen, T. (2018). Inclusive Work Practices: Turnover Intentions Among LGBT Employees of the U.S Federal Government. The American Review of Public Administration, 49(4), 482-494. https://doi. org/10.1177/0275074018817376

Sánchez, E. (2017). El movimiento LGBT (I) en Colombia: la voz de la diversidad de género. Logros, retos y desafíos. Reflexión Política, 19(38), 116-131 https://www.redalyc.org/pdf/110/11054032009. pdf

Swan, L., Henry, R., Smith, E., Aguayo Arelis, A., Rabago Barajas, B., \& Perrin, P. (2019). Discrimination and Intimate Partner Violence Victimization and Perpetration Among a Convenience Sample of LGBT Individuals in Latin America. Journal of Interpersonal Violence. https://doi.org/10.1177/0886260519844774 